\title{
CORRIGENDUM
}

\section{Targeting prohibitins with chemical ligands inhibits KRAS-mediated lung tumours}

H Yurugi, F Marini, C Weber, K David, Q Zhao, H Binder, L Désaubry and K Rajalingam

Oncogene (2017) 36, 5914; doi:10.1038/onc.2017.307; published online 28 August 2017

Correction to: Oncogene (2017) 36, 4778-4789; doi:10.1038/onc. 2017.93; published online 17 April 2017

Following the publication of this article the authors noted that the description of Figure $4 \mathrm{a}$ and Supplementary Figure S2 needed to be corrected for appropriateness in the main text. The passage on page 2 of the PDF was consequently amended to read:
'We then tested if rocaglamide treatment could inhibit KRAS-GTP in cells carrying mutated KRAS. Interestingly, treatment of both 482T1 (Supplementary Figure S2A) and $\mathrm{NCl}-\mathrm{H} 226$ cells (wild type KRAS) with rocaglamide led to inhibition of RAS-GTP loading in these cells in a concentration-dependent manner (Figure $4 \mathrm{a}$ and Supplementary Figure S2B). We then checked...'

The authors apologise for any inconvenience this might have caused. 